\title{
Research on Sliding Mode Speed Control of Permanent Magnet Synchronous Motor Based on MRAS Title
}

\author{
Suying Zhang ${ }^{a}$, Yuelong Wang ${ }^{b, *}$, Huixian Liu, Yue Meng, Mingzhao Li \\ School of Electrical Engineering, Hebei University of Science and Technology, Shijiazhuang \\ a zhsy8985@163.com, bhbkj_28@126.com \\ YYuelong Wang
}

\begin{abstract}
The traditional permanent magnet synchronous motor (PMSM) control system uses a mechanical sensor to detect the speed of the motor and the position of the rotor, the sensor is susceptible to the environment and reduces the reliability of the system. This paper uses a model reference adaptive system (MRAS) instead of a mechanical sensor to estimate the speed of the motor and the position of the rotor.Taking the mathematical model of the three-phase permanent magnet synchronous motor as a reference model,taking the motor speed as the identification parameter to establish an adjustable model,the reference adaptive law is determined according to Popov's hyperstability theorem. The speed controller of the control system adopts sliding mode control (SMC) based on integral sliding surface and exponential reaching law. The simulation results show that the sliding speed control system based on MRAS for permanent magnet synchronous motor can realize the estimation of the motor speed and rotor position, which can guarantee the good dynamic quality of the speed control system.
\end{abstract}

Keywords: permanent magnet synchronous motor,sliding-mode control,model refere- -nce adaptive system

\section{Introduction}

The traditional permanent magnet synchronous motor control system uses a mechanical sensor to detect the speed of the motor and the position of the rotor. The commonly used mechanical sensors increase the volume of the system and the rotary inertia of the rotor, increase system cost, and reduce system reliability ${ }^{[1]}$. Without speed and position sensor technology becomes more and more important.Commonly used methods include estimation methods based on various observers, model reference adaptation, high-frequency signal injection methods, and artificial intelligence estimation methods ${ }^{[2]}$.The observer-based estimation method needs to reconstruct a system, and the input is a measurable variable of the original system. When a certain condition is reached, the output is equivalent to the original system state.Among them, the Full-order State Observer is greatly affected by changes in motor parameters,Sliding Mode Observer causes jitter in the system due to the discontinuity of sliding mode variable structure control,Extended Kalman Filter algorithm is complex and computationally intensive.The high-frequency signal injection method uses the spatial salient pole effect of the motor, but the surface-mounted permanent magnet synchronous motor has no salient pole effect and the control effect is general.The Model Reference Adaptive method is simple in structure and can guarantee the asymptotic convergence of parameter estimation. Due to the closed-loop observation model, the estimation accuracy is high and the anti-interference is strong.

Sliding Mode Control is a kind of special nonlinear control strategy,due to its simple controller design, fast response and strong robustness to parameter variations and external disturbances, it is widely used $^{[3]}$. Therefore, the speed controller adopts sliding mode control. Using the integral sliding mode surface function that add the integration variable of quantity of state to the normal sliding 
surface can effective eliminate steady-state error.An exponential approach law is used to suppress the inherent buffeting of sliding mode control.The simulation results show that the controller has fast response speed, high precision, strong robustness, and can ensure good speed regulation performance of the system.

\section{Mathmatical Model of Permanent Magnet Synchronous Motor}

Since there is no problem with asynchronous motor slip,the vector control of the three-phase permanent magnet synchronous motor is more convenient to implement.Common vector control methods include the control of $i_{d}=0$ and maxim- -um torque per ampere control, the control of $i_{d}=0$ is more suitable for the surface mount type three-phase permanent magnet synchronous motor in this paper.In order to facilitate the design of the controller, the mathematical model of the synchronous rotation coordinate system is selected, and the stator voltage equation can be express-ed as (1).

$$
\left\{\begin{array}{l}
u_{d}=R i_{d}+L_{d} \frac{d i_{d}}{d_{t}}-n_{p} \omega_{m} L_{q} i_{q} \\
u_{q}=R i_{q}+L_{q} \frac{d i_{q}}{d_{t}}+n_{p} \omega_{m} L_{d} i_{d}+n_{p} \omega_{m} \psi_{f} \\
J \frac{d \omega_{m}}{d t}=T_{e}-T_{L}-B \omega_{m} \\
T_{e}=\frac{3}{2} n_{p} \psi_{f} i_{q}
\end{array}\right.
$$

(1)

In the formula, $u_{d}$ and $u_{q}$ are the stator voltages for the $d$ and $q$ axes; $i_{d}$ and $i_{q}$ are the stator currents for the $d$ and $q$ axes; $L_{d}$ and $L_{q}$ are the stator inductances for the $d$ and $q$ axes(For surface mount type three-phase permanent magnet synchro- -nous motor,there are $L_{d}=L_{q}=L$ ); $R$ is the stator resistance; $n_{p}$ is the pole pair number; $\omega_{m}$ is the rotor mechanical angular velocity; $\psi_{f}$ is the permanent magnet flux linkage; $J$ is the moment of inertia; $T_{e}$ is the electromagnetic torque; $T_{L}$ is the load torque ${ }^{[4]}$.

\section{Sliding-Mode Speed Controller Design}

Sliding mode control is a kind of variable structure control system.The essential difference with conventional control is the discontinuity of control. The structure of the system presents the switching characteristics over time, that is, the structure of the control system makes small amplitude and high frequency switching movement according to the preset state trajectory ${ }^{[5]}$. This kind of motion can be designed, and it is not affected by the system parameters and disturbances,making the system has a good robustness.

State variables of three-phase permanent magnet synchronous motor.

$$
\left\{\begin{array}{l}
x_{1}=\omega_{r}-\omega_{m} \\
x_{2}=\int_{0}^{t} x_{1} d t
\end{array}\right.
$$

$\omega_{r}$ is the artificially set motor reference speed; $\omega_{m}$ is the actual speed of the motor.Derivative with respect to (2). 


$$
\left\{\begin{array}{l}
\dot{x}_{1}=-\frac{d \omega_{m}}{d t}=-\frac{3 n_{p} \psi_{f} i_{q}}{2 J}+\frac{T_{L}}{J}+\frac{B \omega_{m}}{J} \\
\dot{x}_{2}=x_{1}
\end{array}\right.
$$

The introduction of the integral term of the velocity error in the conventional sliding surface can improve the speed adjustment accuracy and ensure the robustness of the system. The sliding mode surface $\mathrm{s}$ is (4).

$$
s=x_{1}+c x_{2}=x_{1}+c \int_{0}^{t} x_{1} d t
$$

Taking the derivative of the derivative (5).

$$
\dot{s}=\dot{x}_{1}+c \dot{x}_{2}=-\frac{3 n_{p} \psi_{f} i_{q}}{2 J}+\frac{T_{L}}{J}+\frac{B \omega_{m}}{J}+c x_{1}
$$

In order to ensure good dynamic quality, the exponential reaching law is used ${ }^{[6]}$.

$$
\dot{s}=-\varepsilon \operatorname{sgn}(s)-k s \quad \varepsilon, k>0
$$

The reference current of 1 axis can be obtained from 5 and 6 , which is 2 .

$$
i_{q}^{*}=\frac{2 J}{3 n_{p} \psi_{f}}\left[\varepsilon \operatorname{sgn}(s)+k s+\frac{T_{L}}{J}+c x_{1}+\frac{B \omega_{m}}{J}\right]
$$

Easy to prove $s \dot{s}<0$, The system is asymptotically stable under the action of sliding mode speed controller.

\section{Position Sensorless Control Method for Permanent Magnet Synchronous Motor Based on MRAs}

The model reference adaptive system consists of a reference model with no unknown parameters and an adjustable model with parameters to be estimated. There is a difference in the outputs of the two models, but the physical meaning is the same. In the process of the adaptive law of parameters makes the generalized error of the two models approach zero, the estimated parameters of the adjustable model will converge to the actual values of the reference model. There are three ways to establish a parameter adaptive law ${ }^{[7]}$.Comprehensive comparison of the three methods, This paper adopts the design method based on the theory of super-stability and positive dynamic system ${ }^{[8]}$.

\subsection{Determination of Reference Model and Adjustable Model}

By sort out (2) can obtain the current equation form of the three-phase permanent magnet synchronous motor.

$$
\left\{\begin{array}{l}
p i_{d}=-\frac{R}{L} i_{d}+\omega_{e} i_{q}+\frac{1}{L} u_{d} \\
p i_{q}=-\frac{R}{L} i_{q}+\frac{1}{L} u_{q}-\omega_{e}\left(i_{d}+\frac{\psi_{f}}{L}\right)
\end{array}\right.
$$

The state space expression after the equivalent transformation of (8) is made by reference (9) is (10).

$$
p\left[\begin{array}{l}
i_{d}^{*} \\
i_{q}^{*}
\end{array}\right]=\left[\begin{array}{cc}
-\frac{R}{L} & \omega_{e} \\
-\omega_{e} & -\frac{R}{L}
\end{array}\right]\left[\begin{array}{l}
i_{d}^{*} \\
i_{q}^{*}
\end{array}\right] \frac{1}{L}\left[\begin{array}{l}
u_{d}^{*} \\
u_{q}^{*}
\end{array}\right]
$$

Namely

$$
p i^{*}=A i^{*}+B u^{*}
$$

Where $p$ is a differential operator, and $p=\frac{d}{d t}, i_{d}{ }^{*}=i_{d}+\frac{\psi_{f}}{L}, i_{q}{ }^{*}=i_{q}, u_{d}{ }^{*}=u_{d}+\frac{R}{L} \psi_{f}, u_{q}{ }^{*}=u_{q}$

Change formula (10) to estimate form. 
Namely

$$
p\left[\begin{array}{l}
\hat{i}_{d}^{*} \\
\hat{i}_{q}^{*}
\end{array}\right]=\left[\begin{array}{cc}
-\frac{R}{L} & \hat{\omega}_{e} \\
-\hat{\omega}_{e} & -\frac{R}{L}
\end{array}\right]\left[\begin{array}{l}
\hat{i}_{d}^{*} \\
\hat{i}_{q}^{*}
\end{array}\right]+\frac{1}{L}\left[\begin{array}{l}
u_{d}^{*} \\
u_{q}^{*}
\end{array}\right]
$$

(10) is the reference model, (12) is the adjustable model, and $\hat{\omega}_{e}$ is the speed parameter to be identified.

\subsection{Determination of The Reference Adaptive Law}

Let $e=i^{*}-\hat{i}^{*}$ be the system state generalized error.The difference between the reference model(10)and the adjustable model(9)is(14).

$$
p e=A e-(\hat{A}-A) \hat{i}^{*}
$$

The stability of the system must satisfy two conditions of the Popov's hyperstability theorem ${ }^{[9]}$

(1) The linear time-invariant link is a strictly positive definite matrix.

(2) $\eta\left(0, t_{1}\right)=\int_{0}^{t_{1}} V^{T} W d t \geq-\gamma_{0}^{2}, \forall t_{1} \geq 0, \gamma_{0}$ is any finite positive number.

In order to prove the two conditions, formula (14) is rewritten as follows.

$$
\left\{\begin{array}{l}
p e=A e-B W \\
V=C e
\end{array}\right.
$$

In formula $(15) B=I, C=I, W=(\hat{A}-A) \hat{i}^{*}$.

It is easy to prove that $G(S)=C(S I-A)^{-1}$ is a strictly positive definite matrix, The inverse solution to the popov integral inequality can obtain the adaptive law,Sub--stituting $V, W$ into the inequality of the second condition of Popov's hyperstability theorem.

$$
\eta\left(0, t_{1}\right)=\int_{0}^{t_{1}} e^{T}\left(\omega_{e}-\hat{\omega}_{e}\right) J \hat{i}^{*} d t
$$

$J=\left[\begin{array}{cc}0 & -1 \\ 1 & 0\end{array}\right]$, The estimated form of $\hat{\omega}_{e}$ is taken as the proportional integral form.

$$
\hat{\omega}_{e}=\int_{0}^{t} f_{1}(v, t, \tau) d \tau+f_{2}(v, t)+\hat{\omega}_{e}(0)
$$

(16) and (17) combined to get (18)

$$
\eta\left(0, t_{1}\right)=\int_{0}^{t_{1}}-e^{T}\left[\int_{0}^{t} f_{1}(v, t, \tau) d \tau+f_{2}(v, t)+\hat{\omega}_{e}-\omega_{e}\right] J \hat{i}^{*} d t
$$

Order $-e^{T}=E, \eta\left(0, t_{1}\right)=\eta_{1}\left(0, t_{1}\right)+\eta_{2}\left(0, t_{1}\right)$. You can get the following two formulas.

$$
\begin{gathered}
\eta_{1}\left(0, t_{1}\right)=\int_{0}^{t_{1}} E\left[\int_{0}^{t} f_{1}(v, t, \tau) d \tau+\hat{\omega}_{e}-\omega_{e}\right] J \hat{i}^{*} d t \\
\eta_{2}\left(0, t_{1}\right)=\int_{0}^{t_{1}} E f_{2}(v, t) J \hat{i}^{*} d t
\end{gathered}
$$

Combined with (19),we can use inequality (21) to get (22).

$$
\begin{gathered}
\int_{0}^{t_{1}} \dot{f}(t) k f(t)=\frac{k}{2}\left[f^{2}\left(t_{1}\right)-f^{2}(0)\right] \geq \frac{1}{2} k f^{2}(0) \\
f_{1}(v, t, \tau)=k_{i} E J \hat{i}^{*} \quad\left(k_{i}>0\right)
\end{gathered}
$$

At the same time $\eta_{1}\left(0, t_{1}\right) \geq-\gamma_{1}^{2}$ is proven.

Let $f_{2}(v, t)=k_{p} E J \hat{i}^{*} \quad\left(k_{p}>0\right)$ then $\eta_{2}\left(0, t_{1}\right) \geq-\gamma_{2}^{2}$. The inequality of the second condition of the Popov super-stability theorem is workable.In turn, it can prove that the formula for the proportional integral of $\hat{\omega}_{e}$ is workable.

Sort and merge

$$
\hat{\omega}_{e}=\int_{0}^{t} k_{i} E J \hat{i}^{*} d \tau+k_{p} E J \hat{i}^{*}+\hat{\omega}_{e}(0)
$$




$$
\hat{\omega}_{e}=\left(\frac{K_{i}}{s}+K_{p}\right)\left[i_{d} \hat{i}_{q}-\hat{i}_{d} i_{q}-\frac{\psi_{f}}{L}\left(i_{q}-\hat{i}_{q}\right)\right]
$$

The estimated value of rotor position is obtained by integrating the estimation of motor speed.

$$
\hat{\theta}_{e}=\int \hat{\omega}_{e} d \tau
$$

\section{Simulation Verification}

The system adopts vector control of $i_{d}=0$ to simulate under Simulink. The parameters of permanent magnet synchronous motor are as follows:Stator resistance $R=2.875 \Omega$, Inductance $L=8.5 \mathrm{mH}$, Coefficient of viscosity $B=0$, Rotary inertia $J=0.0008$, Rotor flux $\psi_{f}=0.175 \mathrm{~Wb}$. The simulation model is shown in Figure 1.

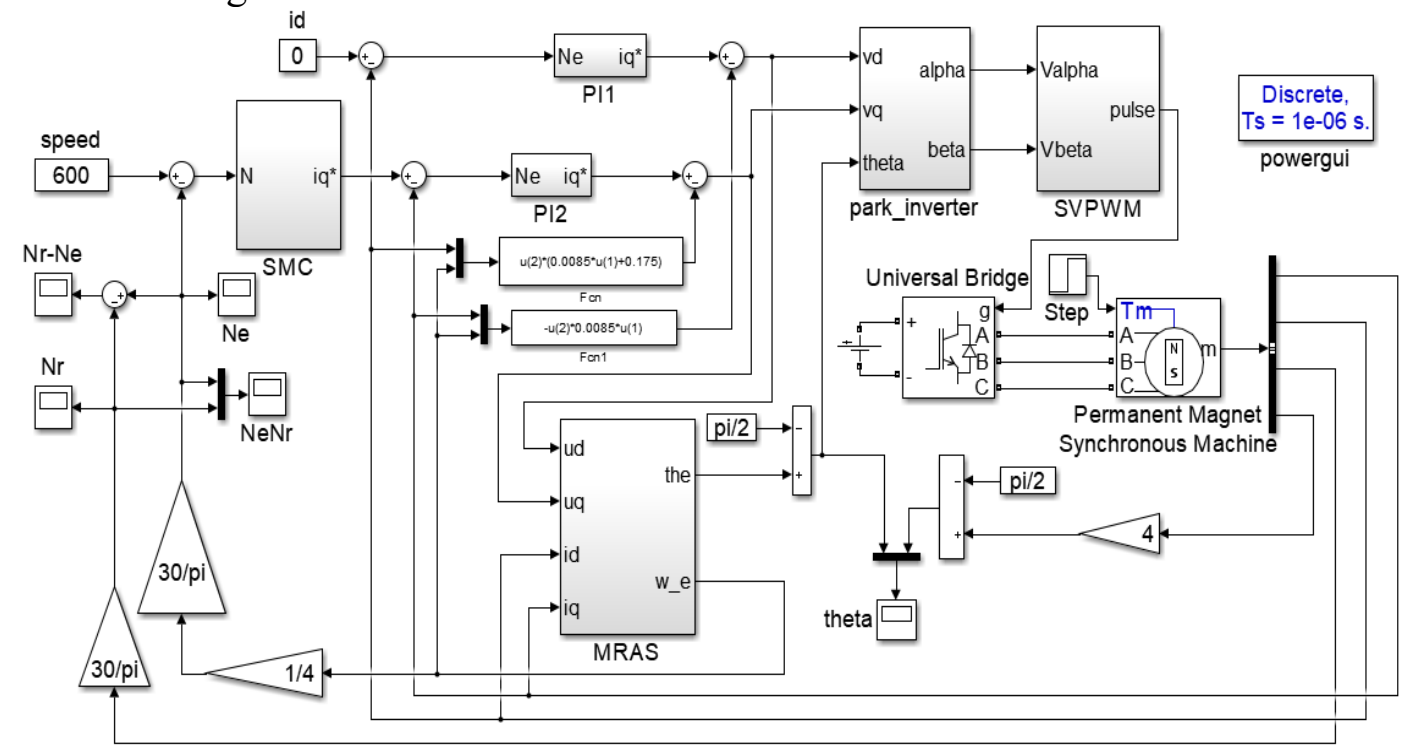

Fig.1 Simulation model of sliding mode speed control system for permanent magnet synchronous motor based on MRAS

The reference speed is $600 \mathrm{r} / \mathrm{min}$, the starting load is $5 \mathrm{~N}$, and the load becomes $10 \mathrm{~N}$ at $0.2 \mathrm{~s}$. The velocity diagram of the motor under the action of the sliding mode speed controller when there is a position speed sensor is shown in FIG. 2 .As can be seen from the figure, under the action of the sliding mode speed controller, the motor rotation speed is almost free of overshoot, the response is fast, and the load disturbance can be well suppressed and quickly return to a stable state.

All the conditions are unchanged, and the MRAS is substituted for the position speed sensor. The speed diagram of the motor under the action of the sliding mode speed controller is shown in figure 3.The error between the actual speed and the estimated speed is shown in Fig. 4. The actual position angle and the estimated position angle are shown in Fig. 5. As can be seen from figure 3 and figure 4 , the actual value of the motor speed is basically coincident with the estimated value at $0.05 \mathrm{~s}$, and the error is almost zero. When the load changes at $0.2 \mathrm{~s}$, under the action of the sliding mode speed controller, the motor speed can be rapidly tuned back to coincide with the estimated speed again.It can be seen from figure 5 that the actual position Angle and the estimated position Angle of the rotor can also reach the basic no-error. 

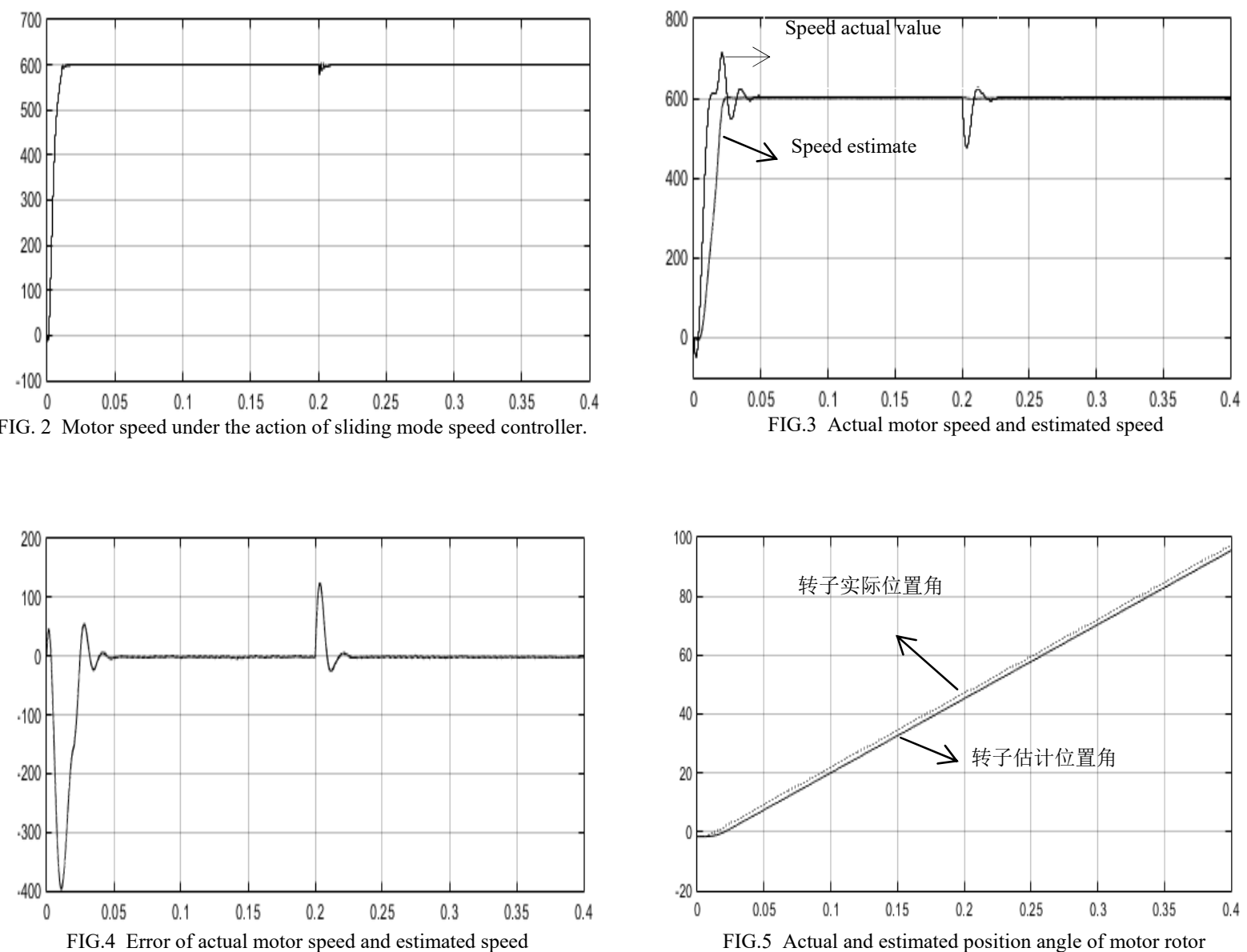

\section{Conclusion}

For the issues that mechanical sensors are susceptible to environmental impact and reducing system reliability.This paper designs a control system based on MRAS for permanent magnet synchronous motor without position and speed sensor and adopts sliding mode speed controller to adjust speed.Simulation experiments verify that SMC has fast response, small overshoot, and strong anti-interference ability.It also verified that MRAS can effectively detect the motor speed and rotor position angle. The whole system can realize the control of permanent magnet synchronous motor when there is no speed and position sensor.

\section{Acknowledgments}

This work was financially supported by the National Natural Science Foundation of China(Grant No. 51507048).

\section{References}

[1] A S Mohamed,M S Zaky,S Z Ashraf,et al. Comparative study of sensorless control methods of PMSM drives[J]. Innovative Systems Design and Engineering,2011,2(5):44-67.

[2] YUAN Lei,SHEN Jianqing,XIAO Fei,CHEN Mingliang. Nonsingular terminal sliding-mode observer design for interior permanant magnet synchronous motor drive at very low-speed[J]. Acta Physica Sinica,2013,62(3):030501. 
[3] ZHANG Xiaoguang, ZHAO Ke, SUN Li, AN Quntao. A PMSM Sliding Mode Control System Based on A Novel Reaching Law[J]. Proceedings of the CSEE,2011,31(24):77-82. [4] YANG Shusheng, ZHONG Yisheng. Robust Controller Design for PMSM Speed Servo Systems[J]. Proceedings of the CSEE,2009,29(3):84-89.

[5] LIU Jian,SU Mei,PEI Mao-lin, ZHANG Chunqiang. TSMC-PMSM Vector Control System Based on New Rate Reaching Law Sliding Mode Controller[J]. MICROMOTORS,2012(11):48-51. [6] W.Gao and J.Hung. Variable structure control of nonlinear systems:a new approach[J]. IEEE Transactions on Industrial Electronics, 1993,40(1):45-55.

[7] LI Yanjun,ZHANG Ke. Adaptive Control Theory and Application[M]. Xi An: Northwestern Polytechnical University Press,2005.

[8] WANG Chengyuan,XIA Jiakuan,YANG Junyou,SUN Yibiao. Modern Motor Control Technology[M]. Peking:CHINA MACHINE PRESS,2006.

[9] XU Qiang,JIA Zhengchun,LI Langru. A Speed Controller of Permanent Magnet Synchronous Motor Using Mras[J]. DIANQI CHUANDONG,1998,(5):21-25. 